\title{
Protecting Breastfeeding during the COVID-19 Pandemic
}

Ritu Cheema, MD ${ }^{1}$ Elizabeth Partridge, MD, MPH ${ }^{1}$ Laura R. Kair, MD, MAS ${ }^{2}$ Kara M. Kuhn-Riordon, MD ${ }^{3}$ Angelique I. Silva, MS, RNC-OB, C-EFM ${ }^{4}$ Maria E. Bettinelli, MD, IBCLC 5 Caroline J. Chantry, MD 6 Mark A. Underwood, $\mathrm{MD}^{3}$ Satyan Lakshminrusimha, $\mathrm{MD}^{3}$ Dean Blumberg, $\mathrm{MD}^{1}$

1 Division of Pediatric Infectious Disease, Department of Pediatrics, University of California, Davis, School of Medicine, Sacramento, California

${ }^{2}$ Division of Pediatric Hospital Medicine, Department of Pediatrics, University of California, Davis, School of Medicine, Sacramento, California

${ }^{3}$ Division of Neonatology, Department of Pediatrics, University of California, Davis, School of Medicine, Sacramento, California

${ }^{4}$ Women's Pavilion and Newborn Nursery, UC Davis Medical Center, Sacramento, California

${ }^{5}$ Department of Clinical Science and Community Health, University of Milan, Milan, Italy

${ }^{6}$ Division of General Pediatrics, Department of Pediatrics, University of California, Davis, School of Medicine, Sacramento, California
Address for correspondence Ritu Cheema, MD, Division of Pediatric Infectious Diseases, Department of Pediatrics, University of California, School of Medicine, UC Davis Children's Hospital, Sacramento, CA-95817 (e-mail: ritcheema@ucdavis.edu).

Am J Perinatol 2023;40:260-266.

\section{Abstract \\ Keywords \\ - SARS-CoV-2 \\ - COVID-19 \\ - breastfeeding \\ - breast milk \\ - maternal-baby separation}

The severe acute respiratory syndrome-coronavirus-2 (SARS-CoV-2) pandemic has impacted all patient populations including pregnant mothers. There is an incomplete understanding of SARS-CoV-2 pathogenesis and transmission potential at this time and the resultant anxiety has led to variable breastfeeding recommendations for suspected or confirmed mothers with novel coronavirus disease 2019 (COVID-19). Due to the potential concern for transmission of infection from maternal respiratory secretions to the newborn, temporary separation of the maternal-baby dyad, allowing for expressed breast milk to be fed to the infant, was initially recommended but later revised to include breastfeeding by the American Academy of Pediatrics in contrast to international societies, which recommend direct breastfeeding. This separation can have negative health and emotional implications for both mother and baby. Only two publications have reported SARS-CoV-2 in human breast milk but the role of breast milk as a vehicle of transmission of COVID-19 to the newborns still remains unclear and may indeed be providing protective antibodies against SARS-CoV-2 infection even in infected neonates. Other modes of transmission of infection to neonates from infected mothers or any care providers cannot be overemphasized. Symptomatic mothers on hydroxychloroquine can safely breastfeed and no adverse effects were reported in a baby treated with remdesivir in another drug trial. The excretion of sarilumab in human breast milk is unknown at this time. Hence, given the overall safety of breast milk and both short-term and long-term nutritional, immunological, and developmental advantages of breast milk to newborn, breast milk should not be withheld from baby. The setting of maternal care, severity of maternal infection and availability of resources can received

June 5,2020

accepted

June 18, 2020

article published online

April 20, 2021 (c) 2021. Thieme. All rights reserved.

Thieme Medical Publishers, Inc.,

333 Seventh Avenue, 18th Floor,

New York, NY 10001, USA
DOI https://doi.org/

10.1055/s-0040-1714277.

ISSN 0735-1631. 
impact the decision of breastfeeding, the role of shared decision making on breastfeeding between mother and physician needs to be emphasized. We strongly recommend direct breastfeeding with appropriate hygiene precautions unless the maternal or neonatal health condition warrants separation of this dyad.

\section{Key Points}

- Breastmilk does not appear to play a significant role in transmission of SARS-CoV-2.

- Mother-baby separation has negative health and emotional consequences.

- Mothers with suspected or confirmed COVID-19 can directly breastfeed with appropriate precautions.

On March 11, 2020, the World Health Organization (WHO) declared novel coronavirus disease 2019 (COVID-19) a pandemic with more than a million confirmed cases. ${ }^{1}$ The health impact of severe acute respiratory syndrome-coronavirus-2 (SARS-CoV-2) spans across all age groups including pregnant mothers. For mothers and their newly born infants, breastfeeding during this pandemic deserves special attention due to its short and long-term health implications. In the United States, uncertainty and social anxiety about COVID-19 is likely driving the practice of maternal-baby dyad separation and limiting breastfeeding, especially in hospitals located in areas with high community prevalence. The aim of this article is to provide guidance to clinicians and families on breastfeeding in the context of SARS-CoV-2.

Several international health agencies and medical societies including the $\mathrm{WHO},{ }^{2}$ Royal College of Obstetricians and Gynaecologists (RCOG), ${ }^{3}$ and Italian Society of Neonatology $(\mathrm{SIN})^{4}$ support direct breastfeeding with hygiene precautions and recommend mother and neonate separation only in situations of poor maternal health conditions or the need to provide therapies to the newborn. In contrast, U.S. agencies, including the Centers for Disease Control and Prevention (CDC) and the American Academy of Pediatrics (AAP), had taken a more conservative approach to potential maternal-child transmission. Revised guidelines published by AAP subsequently promote both direct breastfeeding and use of expressed breastmilk. The CDC recognizes the importance of mother-baby contact and shared decision making between family and physician but does not give clear guidance on breastfeeding. ${ }^{5}$ The AAP recommends temporary separation of mother-infant dyads and administration of expressed breast milk rather than direct breastfeeding to decrease the risk of postnatal infant infection from maternal respiratory secretions. ${ }^{6}$ In U.S. medical centers following CDC and AAP guidance, we see routine separation of babies from mothers with possible or suspected SARS-CoV-2 infections. In these situations, only a limited number of maternal-baby dyads whose mothers request direct breastfeeding receive the benefits of maternal-infant contact. Separated dyads experience the potentially harmful effects of separation as shown in -Fig. 1. A vicious cycle of separation-induced stress, reduced breast milk production, poor breast feeding success, and loss of benefits of skin-to-skin care increase the risk of infant instability and the potential need for neonatal intensive care unit (NICU) care as a result of infant separation from the mother. - Fig. 1 represents the negative effects of separation of mother and infant as outlined by Stuebe in a recent editorial. $^{7}$

In the setting of the conflicting recommendations between international and U.S. national societies, our institution(UC Davis Children's Hospital) has developed two practical approaches to human milk feeding based on severity of maternal illness with COVID-19 and the mother's ability to breastfeed. Several factors including setting of maternal care, severity of maternal infection, and availability of resources may play a role in breastfeeding decisions during the COVID-19 pandemic. Additionally, the decisions about breastfeeding and provision of human milk require weighing of the benefits including the nutritional, immunological, and developmental advantages of human milk and emotional bonding from breast feeding, and the risk of infection to the newborn. ${ }^{8-10}$ It is important to understand the epidemiology of SARS-CoV-2, risk of transmission of infection from mother to newborn, and severity of infection in newborns to analyze the risk versus benefit of direct breastfeeding to the baby.

\section{Risk, Incidence, and Severity of Infection in the Newborn}

There is no data to inform whether pregnancy increases susceptibility to COVID-19.There are several emerging viruses including Zika, Ebola, and Marburg that can be transmitted in utero. COVID-19 does not appear to share this pattern of transmission. While maternal morbidity during the 2002 to 2003 SARS-CoV epidemic was well documented, there was no evidence of vertical transmission among live newborn infants. Although studies were limited, no viral RNA was detected by RT-PCR (reverse transcription polymerase chain reaction) in amniotic fluid, placenta, cord blood, or breast milk. Likewise, there are no clinical or serological reports of perinatal transmission with previous SARS and Middle East respiratory syndrome (MERS) epidemic. ${ }^{11}$ At this point in the global pandemic of COVID-19, scattered reports of miscarriage have been published including one in which SARS-CoV-2 was detected in placental tissue but not detected in the stillborn infant born to a symptomatic mother with COVID-19 infection. ${ }^{12}$ Unlike the mosquitoborne Zika virus which can be passed from a pregnant woman to her fetus leading to birth defects, there is no evidence at this time that SARS-CoV-2 is transmitted to the 


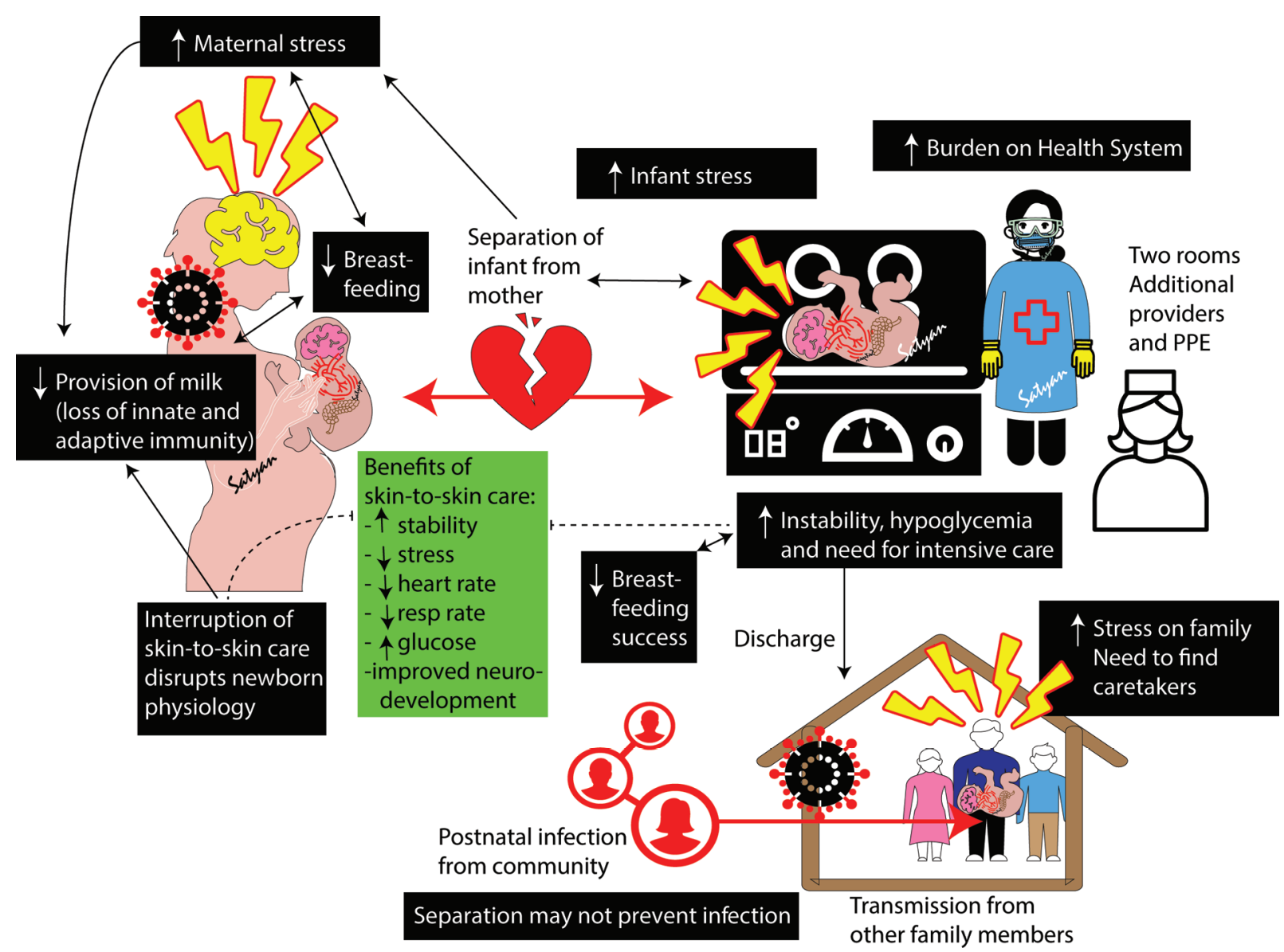

Fig. 1 Negative effects of separation of a mother who is confirmed or suspected with SARS-CoV-2 infection (Image copyright Satyan Lakshminrusimha). SARS-CoV-2, severe acute respiratory syndrome-coronavirus-2.

Copyright Satyan Lakshminrusimha. Readers are welcome to use this infographic as posters, slides, or brochures for patient, parent, and trainee education.

fetus or the newborn. In a review of 38 pregnant women from China with acute COVID-19 in the third trimester of pregnancy, SARS-CoV-2 was not detected in amniotic fluid, breastmilk, or umbilical cord blood samples tested. Among the 30 neonates delivered by these women, who underwent testing, none were positive for SARS-CoV-2 by RT-PCR. ${ }^{13}$ In another report describing clinical outcomes in 10 neonates born to mothers with SARS-CoV-2 pneumonia, some babies developed symptoms of shortness of breath (6), cyanosis (3), gastric bleeding (2), and 1 baby died of multiple organ failure and disseminated intravascular coagulation. Throat swabs from all those infants were negative for SARS-CoV-2, suggesting that these neonatal complications were not related to intrauterine transmission. ${ }^{14}$

Recent reports of neonates with elevated immunoglobulin (Ig)-M antibodies to SARS-CoV-2 drawn following birth have raised the possibility of in utero transmission. However, all those neonates were negative for COVID-19 by RT-PCR. ${ }^{15,16}$ Importantly, IgM assays are prone to false-positive and falsenegative results, as well as cross-reactivity, and are usually less reliable than molecular diagnostic tests. This prompts caution in interpreting these results as evidence of vertical transmission. ${ }^{17}$

Studies examining the presence of SARS-CoV-2 in breast milk have been limited. A recent summary made available prior to peer review, reported 9 mothers with confirmed COVID-19 all of whose breast milk samples tested negative for SARS-CoV-2 by RT-PCR. ${ }^{18}$ A recent prepublication (before peer review) by Groß et al reports detection of SARS-CoV-2 in breast milk from a symptomatic mother with confirmed infection whose baby also tested positive for SARS-CoV-2. ${ }^{19}$ In this study, the false positive result from external contamination of breast milk was reportedly ruled but authors concluded the role of breast milk in transmission to baby was unclear. This newborn could have acquired infection through various modes of transmission including from the mother via respiratory route before she started wearing mask, from an infected family member, health care provider, or roommate as the mother shared the room with another SARS-CoV-2 mother during her hospitalization. In another outpatient follow-up study of two neonates, born to the mothers with SARS-CoV-2 infection, both infants tested negative during hospitalization. One of the infants tested positive on day 15 of life but remained asymptomatic which could reflect protection against severe infection from maternal IgG antibodies detected in the baby during hospitalization and possibly from sIgA (secretory immunoglobulin $\mathrm{A}$ ) in breast milk. The second infant remained negative during follow-up despite positive results from placenta, umbilical 
blood, and breast milk (three out of five specimens) during hospitalization. ${ }^{20}$ To our knowledge, these are the only two reports of virus in breast milk. We regard these isolated reports as insufficient evidence to recommend against breastfeeding. In addition, these reports highlight other possible routes of transmission to neonates. Ongoing studies examining breast milk for SARS-CoV-2 virus and antibodies are needed. To date, there are no convincing data suggesting that human milk is a source of SARS-CoV-2 infection. While there is inconclusive evidence of vertical transmission of SARS-CoV-2, there is clear evidence that newborns are susceptible to person-to-person horizontally spread after delivery through close contact with respiratory secretions from an infected symptomatic or asymptomatic caregiver (including mother). ${ }^{21}$

Important to the discussion of breastfeeding risks in the setting of maternal COVID-19 infection is the incidence and severity of COVID-19 in children and neonates. Children have been proportionately less affected compared with adults both in terms of incidence and severity of COVID-19 and thus experience a significantly lower rate of hospitalization. ${ }^{22}$ The CDC recently reported on 149,082 U.S. laboratory-confirmed cases of COVID-19 occurring between February 12 and April 2, 2020. Among the cases where age was known, age group of $<18$ years accounted for 2,572 ; this is $(1.7 \%)$ of all cases, although this age group represents $22 \%$ of the U.S. population. Infants $<1$ year accounted for $15 \%$ of pediatric COVID-19 cases $(n=393)$, and represents $0.27 \%$ of all cases in the United States despite accounting for $1.2 \%$ of the population. $^{23}$ There are several hypotheses for this observation including the relatively low number of angiotensin-converting enzyme (ACE) receptors in younger children (this virus binds to ACE-2 receptors expressed in lungs), immature immune system causing decreased immune response to the viral insult with less immune-dysregulation or cytokine storm, and potential cross-immunity from previous infections with other viruses from the coronavirus family. More studies are needed to further clarify the pathophysiology of SARS-CoV-2 in children, as well as the basis for their decreased rate of infection.

\section{Breast Feeding by Mothers Undergoing Therapy for COVID-19}

For mothers who are receiving treatment for SARS-CoV-2 infection and wish to breastfeed, the infant's exposure to antiviral therapy is a potential concern. Remdesivir, ${ }^{24}$ hydroxychloroquine, and sarilumab ${ }^{25}$ are the most common current therapeutic options under investigation for confirmed COVID19 with remdesivir receiving emergency use authorization on May 1, 2020 from the U.S. Food and Drug Administration (FDA) for adults and children with confirmed COVID-19. While passage of remdesivir to infant via breastmilk is unknown, no adverse events were reported in a newborn whose mother received remdesivir therapy for infection with Ebola. ${ }^{26}$ Furthermore, remdesivir is a large molecular weight (MW) compound $(602.6 \mathrm{~g} / \mathrm{mol})$, significantly limiting its passage into breastmilk. A study of 33 mothers receiving long-term hydroxychloroquine found low levels of the medication in the milk (the infant's weight adjusted relative dose $(\mathrm{mg} / \mathrm{kg}$ of infant weight) was between 1.9 and $3.2 \%$ of the maternal dose in $\mathrm{mg} / \mathrm{kg}$ of maternal weight. ${ }^{27}$ As noted in the National Library of Medicine's LactMed database, infants exposed to hydroxychloroquine during breastfeeding receive only small amounts of this drug in breastmilk. In infants up to at least 1 year of age, careful followup found no adverse effects on growth, vision, or hearing. International experts indicate that hydroxychloroquine is acceptable during breastfeeding. ${ }^{28}$ There are no data available on safety during breastfeeding for sarilumab, an interleukin (IL)-6 IgG1 monoclonal antibody. Sarilumab is also a large protein molecule (MW, 150,000) likely with low levels in human milk; absorption is also unlikely due to potential destruction in the infant's gastrointestinal tract. If sarilumab is required by the mother, the European League Rheumatism (EULAR) task force does not recommend discontinuation of breastfeeding while the NLM database recommends exercising caution until more data are available. ${ }^{29}$

Currently, there is no compelling evidence that suggests transmission of SARS-CoV-2 from mother to baby via vertical route or breast milk. Breast milk is indeed the "elixir of life" for newborns. Breast milk is the ideal mixture of nutrients, cellular components, and bioactive molecules, created by the mother at significant nutritional cost to herself. The health benefits of breast milk for newborns are well described. Of particular, relevance is a possibility that specific IgA with activity against SARS CoV-2 is present in breastmilk from mothers with a history of COVID-19 infection. Indeed, there may be a benefit of providing breast milk from an infected mother to her baby for specific protection against COVID-19, assuming adequate practice of hygiene. As studies of specific serum and milk antibodies become available, the protective value of breast milk and of immunization against SARS-CoV-2 during pregnancy may become clearer. In addition, the benefits of maternal-baby contact in the form of direct breastfeeding cannot be overemphasized. The benefits of breastfeeding in conjunction with the relatively low prevalence of SARS-CoV-2 and mild illness in newborns and children supports the practice of recommending direct breastfeeding as the preferred option during this pandemic. Given the immaturity of the immune system of newborns, they are at risk of acquiring infection from other routes via horizontal transmission from mother or any other care provider or close contact. Thus maintaining appropriate respiratory hygiene when in contact with the newborn remains critical.

\section{Practical Approaches to Human Milk Feeding}

For mothers with suspected or confirmed SARS-CoV-2 infection whose infants are negative or with pending test results, we have outlined two approaches to breast milk feeding. Shared decision making between provider and mother is a critical component in choosing optimal nutrition for these infants. ${ }^{30}$ The main guiding principles are (1) the benefits of breast feeding for both members of the dyad, (2) risk of transmission from mother to neonate, and (3) expected relative mild severity of disease in neonates as discussed 
above. Severity of maternal infection and her ability to directly breastfeed influences this decision-making process, as disease severity relates to viral load, infectivity, and a mother's ability to feed and care for her infant. ${ }^{31}$

\section{Approach to Human Milk Nutrition in Asymptomatic or Mildly Symptomatic Mothers with COVID-19 Able to Directly Breastfeed}

As the risk of transmission from mother to neonate is likely relatively low, and the benefits of maternal-child interaction are maximized with minimal interference to their relationship, direct breast feeding is encouraged ( - Fig. 2). The mother should wear a surgical mask (for source control), wash her hands, and breasts with soap and water before feeds and breastfeed the baby. During intervals between feeds, it is recommended that the infant's crib be placed at least 6 feet ( $2 \mathrm{~m}$ ) from the mother's bed preferably behind a physical barrier (such as a curtain). These precautions continue until mother is afebrile (without antipyretics), demonstrates improvement of symptoms, and has two negative tests for SARS-CoV-2 at least 24 hours apart, resources permitting. If timely testing is not feasible, then these precautions should be discontinued when the mother is symptomatically improved, afebrile for at least 3 days with at least 7 days since the onset of symptoms. In most instances, mother and baby may be discharged home with further testing only if baby demonstrates signs and symptoms of COVID-19.

\section{Approach to Human Milk Nutrition in Moderate to Severely Symptomatic Mothers with COVID-19 Unable to Directly Breastfeed}

In some circumstances, maternal-child direct interaction may be reduced due to maternal inability to breastfeed as a result of her clinical condition (moderate-severe symptoms). In this scenario, the mother washes her breast with soap and water and expresses milk (with a hospital-grade electric breast pump if available) while wearing a mask (-Fig. 3). A healthy family member/nurse feeds the expressed milk to the baby in a separate room. Breast pump tubing and container should be cleaned after each use and mother should preferably have a dedicated breast pump. Strict hand hygiene must be followed. The outside surface of containers must be disinfected after milk expression and before storage, preferably with hospital grade viricidal wipes due to relative stability of this virus on different surfaces to minimize the risk of exposure to health care workers. This approach continues until the mother meets the same criteria as mentioned in - Fig. 2. Importantly, this approach can negatively impact breast feeding and maternal-infant bonding. Hence, the recommendation of direct breastfeeding and the role of shared decision making between mother and physician cannot be overemphasized.

In a scenario, where mothers are in the ICU and very ill or convalescing at home and cannot (or choose not to) perform hand hygiene or wear mask or ensure the cleanliness of the

\section{MATERNAL COVID-19 ASYMPTOMATIC OR MILD ILLNESS}

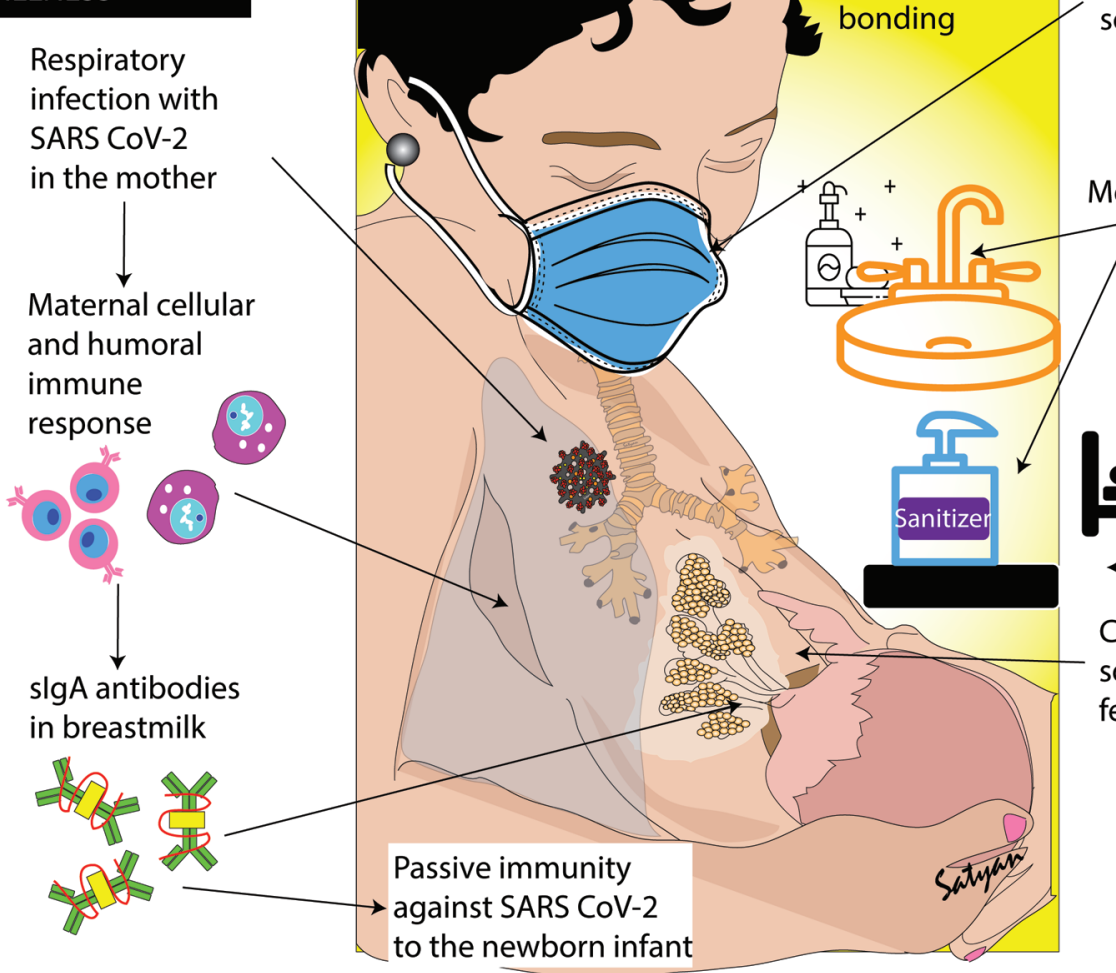

Surgical mask use for source containment

Separation from mother in between feeds with a physical barrier / curtain

Meticulous hand hygiene
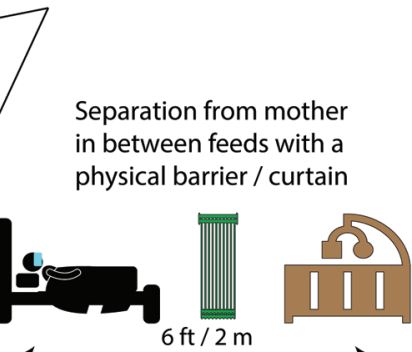

Cleaning the breast with soap and water before feeds

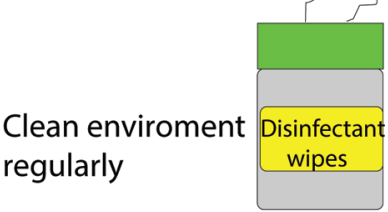

Fig. 2 Approach to human milk nutrition in asymptomatic or mildly symptomatic mothers with COVID-19 able to directly breastfeed. COVID-19, novel coronavirus disease 2019.

Copyright Satyan Lakshminrusimha. Readers are welcome to use this infographic as posters, slides, or brochures for patient, parent, and trainee education. 


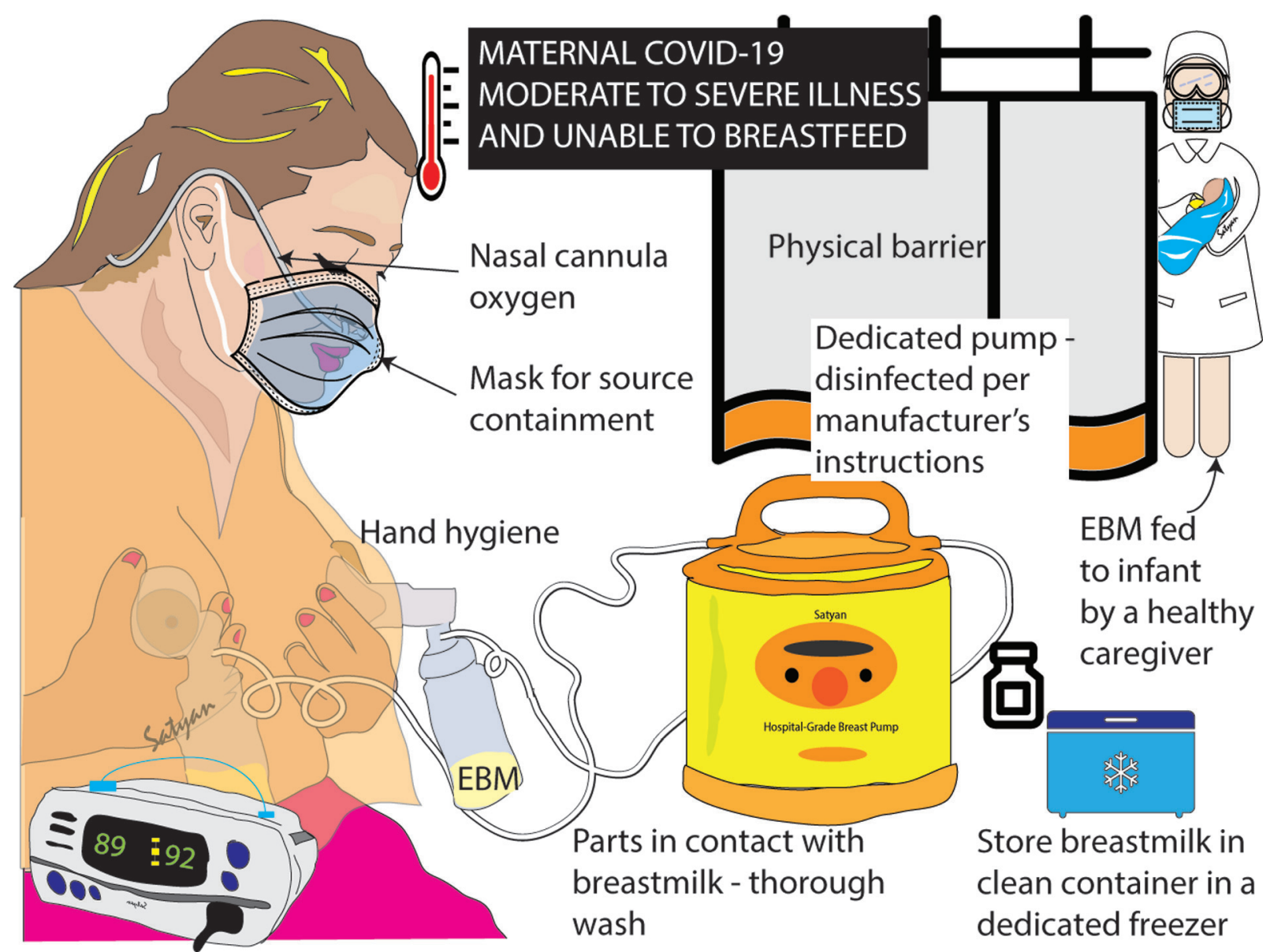

Fig. 3 Approach to human milk nutrition in moderate to severely symptomatic mothers with COVID-19 unable to directly breastfeed. COVID-19, novel coronavirus disease 2019 EBM, expressed breast milk.

Copyright Satyan Lakshminrusimha. Readers are welcome to use this infographic as posters, slides, or brochures for patient, parent, and trainee education.

pumping equipment, pumping for the sole purpose of maintaining supply may be the best temporary option. However, if precautions can be taken to keep baby protected from viral respiratory droplets and keep the breast and milk clean, continuing to feed the infant at the breast is always the preferred option. Every effort should be made to encourage both milk expression and direct breast feeding as early as possible, with potential assistance with expressing breast milk and establishing relactation.

\section{Conclusion}

Considering the benefits of breastfeeding and available data suggesting an insignificant role of human milk in the transmission of SARS-CoV-2 and other coronaviruses, we maintain that mothers with confirmed or suspected COVID-19 can safely breastfeed with appropriate precautions. These recommendations are focused on preparing families for the home environment in the context of widespread community SARS-CoV-2 infection. While this review outlines specific, evidence-based strategies for breastfeeding during the COVID-19 pandemic, further research is needed to understand the role of breast milk in transmission of and protection against SARS-CoV-2.

\section{Funding}

L.R.K. was supported by the National Center for Advancing Translational Sciences, National Institutes of Health (grant: UL1 TR001860), and the National Institutes of Health Building Interdisciplinary Research Careers in Women's Health Program (K12HD051958). The contents of this publication are solely the responsibility of the authors and do not represent the official views of the National Institutes of Health.

\section{Conflict of interest}

None declared.

\section{Acknowledgment}

We thank Dr. Manuela Cardellicchio, obstetrician, ASST FBF Sacco, Milan, for sharing her obstetrical experience during the COVID-19 pandemic.

\section{References}

1 Ghebreyesus TA. WHO Director-General's opening remarks at the media briefing on COVID-19 - 11 March 2020. Available at: https:// www.who.int/dg/speeches/detail/who-director-general-s-opening-remarks-at-the-media-briefing-on-covid-19-11-march-2020. Accessed June 26, 2020 
2 WHO. Breastfeeding advice during the COVID-19 outbreak. Available at: http://www.emro.who.int/nutrition/nutrition-infocus/breastfeeding-advice-during-covid-19-outbreak.html. Accessed April 15, 2020

3 Coronavirus (COVID-19) Infection in Pregnancy: Information for healthcare professionals. Available at: https://www.rcog.org.uk/ en/guidelines-research-services/guidelines/coronavirus-pregnancy/covid-19-virus-infection-and-pregnancy/. Accessed June 26, 2020

4 Davanzo R, Moro G, Sandri F, Agosti M, Moretti C, Mosca F. Breastfeeding and coronavirus disease-2019: Ad interim indications of the Italian Society of Neonatology endorsed by the Union of European Neonatal \& Perinatal Societies. Matern Child Nutr 2020;16(03):e13010

5 CDC. Coronavirus Disease 2019 (COVID-19) Considerations for Inpatient Obstetric Healthcare Settings 2020. Available at: https://www.cdc.gov/coronavirus/2019-ncov/hcp/inpatient-obstetric-healthcare-guidance.html. Accessed April 15, 2020

6 Kumar A. Management of Infants Born to Mothers with COVID19: Initial Guidance for pediatric hospitals. Available at: https:// www.the-hospitalist.org/hospitalist/article/221157/pediatrics/ management-infants-born-mothers-covid-19. Accessed June 26, 2020

7 Stuebe A. Should infants be separated from mothers with COVID19? First, do no harm. Available at: https://www.liebertpub.com/ doi/full/10.1089/bfm.2020.29153.ams. Accessed June 26, 2020

8 Dunne-Castagna VP, Mills DA, Lönnerdal B. Effects of milk secretory immunoglobulin a on the commensal microbiota. Nestle Nutr Inst Workshop Ser 2020;94:158-168

9 Yang B, Chuang H, Chen R. Protection from viral infections by human milk oligosaccharides: direct blockade and indirect modulation of intestinal ecology and immune reactions. Open Glycosci 2012;(05):19-25

10 Victora CG, Horta BL, Loret de Mola C, et al. Association between breastfeeding and intelligence, educational attainment, and income at 30 years of age: a prospective birth cohort study from Brazil. Lancet Glob Health 2015;3(04):e199-e205

11 Schwartz DA, Graham AL. Potential maternal and infant outcomes from (Wuhan) coronavirus 2019-nCoV infecting pregnant women: lessons from SARS, MERS, and other human coronavirus infections. Viruses 2020;12(02):E194

12 Baud D, Greub G, Favre G, et al. Second-trimester miscarriage in a pregnant woman with SARS-CoV-2 infection. JAMA 2020 (e-pub ahead of print) . Doi: 10.1001/jama.2020.7233

13 Schwartz DA. An analysis of 38 pregnant women with COVID-19, their newborn infants, and maternal-fetal transmission of SARSCoV-2: maternal coronavirus infections and pregnancy outcomes. Arch Pathol Lab Med 2020 (e-pub ahead of print) . Doi: 10.5858/ arpa.2020-0901-SA
14 Zhu H, Wang L, Fang C, et al. Clinical analysis of 10 neonates born to mothers with 2019-nCoV pneumonia. Transl Pediatr 2020;9 (01):51-60

15 Dong L, Tian J, He S, et al. Possible vertical transmission of SARSCoV-2 from an infected mother to her newborn. JAMA 2020

16 Zeng H, Xu C, Fan J, et al. Antibodies in infants born to mothers with COVID-19 pneumonia. JAMA 2020

17 Kimberlin DW, Stagno S. Can SARS-CoV-2 infection be acquired in utero?: more definitive evidence is needed JAMA 2020 (e-pub ahead of print) . Doi: 10.1001/jama.2020.4868

18 Lackey KA, Pace RM, Williams JE, et al. SARS-CoV-2 and human milk: what is the evidence? Maternal and Child Nutrition 2020. Doi: $10.1111 / \mathrm{mcn} .13032$

19 Groß R, Conzelmann C, Müller JA, et al. Detection of SARS-CoV-2 in human breast milk. Lancet 2020 (e-pub ahead of print). Doi: 10.1016/S0140-6736(20)31181-8

20 Buonsenso D, Costa S, Sanguinetti M, et al. Neonatal late onset infection with severe acute respiratory syndrome coronavirus 2 . Am J Perinatol 2020

21 Bai Y, Yao L, Wei T, et al. Presumed asymptomatic carrier transmission of COVID-19. JAMA 2020

22 Molloy EJ, Bearer CF. COVID-19 in children and altered inflammatory responses. Pediatr Res 2020

23 CDC COVID-19 Response Team. Coronavirus disease 2019 in children - United States, February 12-April 2, 2020. MMWR Morb Mortal Wkly Rep 2020;69(14):422-426

24 Expanded Access Remdesivir (RDV; GS-5734). Available at: https:// clinicaltrials.gov/ct2/show/NCT04302766. Accessed June 26, 2020

25 Sarilumab COVID-19. Available at: https://clinicaltrials.gov/ct2/ show/NCT04327388. Accessed June 26, 2020

26 Dörnemann J, Burzio C, Ronsse A, et al. First newborn baby to receive experimental therapies survives ebola virus disease. J Infect Dis 2017;215(02):171-174

27 Peng W, Liu R, Zhang L, Fu Q Mei D, Du X. Breast milk concentration of hydroxychloroquine in Chinese lactating women with connective tissue diseases. Eur J Clin Pharmacol 2019;75(11): 1547-1553

28 Hydroxychloroquine, in Drugs and Lactation Database (LactMed). Available at: https://www.ncbi.nlm.nih.gov/books/NBK501150/. Accessed June 26, 2020

29 Sarilumab, in Drugs and Lactation Database (LactMed). Available at: https://www.ncbi.nlm.nih.gov/sites/books/NBK500915/. Accessed June 26, 2020

30 Chandrasekharan P, Vento M, Trevisanuto D, et al. Neonatal resuscitation and postresuscitation care of infants born to mothers with suspected or confirmed SARS-CoV-2 infection. Am J Perinatol 2020

31 Liu Y, Yan LM, Wan L, et al. Viral dynamics in mild and severe cases of COVID-19. Lancet Infect Dis 2020;20(06):656-657 